\title{
Using cross-system diversity in heterogeneous networks: Throughput optimization
}

\author{
Samson Lasaulce ${ }^{\mathrm{a}}$, Alberto Suárez ${ }^{\mathrm{b}, *}$, Raul de Lacerda $^{\mathrm{b}}$, Mérouane Debbah $^{\mathrm{c}}$ \\ ${ }^{a}$ Lab. des Signaux et Systemes, CNRS - SUPELEC - Univ. Paris Sud, 91190, Gif-sur-Yvette, France \\ ${ }^{\mathrm{b}}$ Dpt. Communications Mobiles, Institut Eurecom, 06904, Sophia Antipolis, France \\ ${ }^{\mathrm{c}}$ Chaire Alcatel-Lucent, SUPELEC, 91190, Gif-sur-Yvette, France
}

\section{A R T I C L E I N F O}

\section{Article history:}

Received 19 November 2007

Received in revised form 13 June 2008

Accepted 16 June 2008

Available online 25 June 2008

\section{Keywords:}

Heterogeneous networks

Cross-system diversity

Soft handover

Power allocation

Random matrix theory

Multiple access channel

CDMA

MIMO

OFDM

\begin{abstract}
A B S T R A C T
This paper investigates the situation where a group of terminals can be simultaneously connected to several base stations, using distinct technologies on non-overlapping frequency bands. We introduce and solve the problem of optimal power allocation (in the sense of the ergodic sum-rate), for terminals in the uplink, using three types of receiver: the optimum receiver, minimum mean square error and matched filters. Key results from random matrix theory allow us to solve the corresponding optimization problems. Simulations validate our approach, and illustrate the performance gain obtained by using several technologies simultaneously, instead of one at a time.
\end{abstract}

(c) 2008 Elsevier B.V. All rights reserved.

\section{Introduction}

As the number of wireless systems has increased over the last two decades, the idea of system convergence has been introduced (see e.g. [21,30]), in order to enable mobile terminals to operate with different standards. This convergence idea was one of the driving forces behind the design of reconfigurable terminals, also known as software defined radio, flexible radio [20] or cognitive radio in the context of intelligent terminals [11]. Mobile phones currently available on the market are multi-mode, which means that they can work with different standards. In Europe for example, mobile phones typically have both the GSM, GPRS and/or UMTS-FDD standards implemented, and sometimes even $802.11 \mathrm{a} / \mathrm{b} / \mathrm{g}$ standards via unlicensed mobile access (UMA) technology (see e.g. [14]). In addition, there are many other situations where a terminal can have access to several signals that are in different, non-overlapping frequency bands. Here are a few examples: a GSM mobile station is able to listen to several GSM base stations; a UMTS user equipment can listen to WCDMA base stations, but also possibly TD-CDMA base stations; a DVB-H mobile terminal can operate in the 3G or the DVB standard. In all these examples, the terminal operates with only one standard at a time, depending on the user location and/or the type of service (Internet, TV, voice, ...) requested by the user.

Although the present work is clearly based on an information-theoretic approach, it still provides elements to understand the aforementioned situations, and give some ideas of what could be done to optimize the overall uplink network throughput, by using all the systems simultaneously [19], instead of sequentially (hard handover or best base station

\footnotetext{
* Corresponding author. Tel.: +3349300 81; fax: +33493008180.

E-mail addresses: lasaulce@lss.supelec.fr (S. Lasaulce), alberto.suarez-real@eurecom.fr (A. Suárez), raul.de-lacerda@eurecom.fr (R. de Lacerda), merouane.debbah@supelec.fr (M. Debbah).
} 


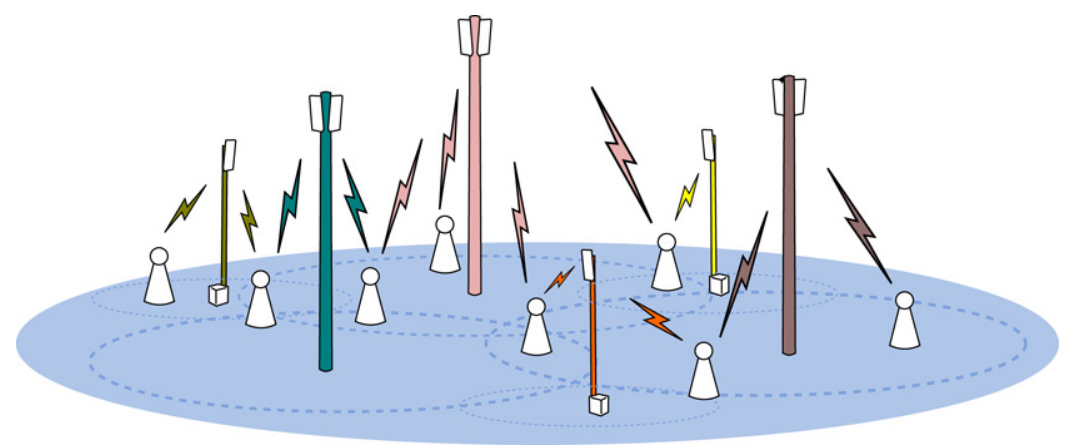

Fig. 1. Cross-system scenario.

selection) as it is the case in existing systems or contributions [10,34]. This will provide an additional form of diversity at the terminals, which could be named cross-system diversity. More specifically, we consider several mobile users and base stations, each of the latter using a particular frequency band and radio access technology. We assume that the base stations are connected through perfect communication links. For instance, in UMTS networks, base stations are connected through a radio network controller and very reliable wired connection (e.g. optic fiber), which is not far from a perfect communication link. Users have wireless links towards the different base stations, and we want to derive the optimal power and rate allocations, given a fixed power constraint for each user. The uplink power allocation (PA) scheme is optimized in order to maximize the sum-rate (over the users and systems) of the overall network.

There exist many works on how to optimally allocate the transmit power to the different sub-channels. To our knowledge, Ref. [18] is the closest work to the one presented here. The authors address the problem of jointly allocating power and subcarriers in the context of orthogonal frequency division multiple access (OFDMA) systems. Our work differs from theirs on several points: we consider a more general channel model (fading channels instead of Gaussian channels), a very different context (heterogeneous networks), all the sub-channels are used whereas in [18], only a subset of them is used by each transmitter and also the optimization problem of [18] is not convex, in contrast with the PA problem for the optimum receiver investigated in this paper. In addition, our main goal is to optimize a global performance criterion under local power constraints. Finally, our information theoretic approach exploits asymptotic random matrix theory [13,31], in order to provide tractable expressions for the optimization problems under investigation. Hence, we will assume the dimensions of the systems as well as the number of users large enough, in order to benefit from the self-averaging properties of the matrices under consideration. In particular, an interesting feature of these self-averaging properties shows that only the parameters of interest to the problem (system load, signal to noise ratio,...) are kept, whereas all irrelevant parameters disappear $[15,22,27,28]$. This provides a neat analysis framework for multi-dimensional problems. Moreover, although the results are proved in the asymptotic regime, it turns out (due to fast convergence properties) that they are accurate even for rather small systems (see e.g. $[2,7,8,25]$ ).

This paper is structured as follows. Section 2 provides the signal model used to study the cross-system problem under investigation. In Section 3, we consider the case where the receiver is equipped with the information theoretic optimum decoder. ${ }^{1}$ In order to gain insight into the overall sum-capacity maximization problem we start by studying the simplest scenario (Section 3): one single user, two one-dimensional base stations with equal bandwidths and the different links assumed to be static. In Section 4, the sum-capacity achieving power allocation policy is provided in a general framework: arbitrary numbers of users, base station dimensions, systems bandwidths and fading channels with transmit and receive correlations. As a second step we investigate more realistic receiver structures in terms of complexity, namely the minimum mean square error (MMSE) receiver and the matched filter (MF), optimizing the overall system sum-rate achieved when the base stations use these receivers. Simulations are provided in Section 5 to assess the gain provided by the proposed approach and possible extensions of this work are given in Section 6.

Notations: in this paper, the notations $s, \underline{v}$, $\mathbf{M}$ stand for scalar, vector and matrix respectively. Capital letters are used to denote index upper bounds. The superscripts $(.)^{\mathrm{T}}$ and $(.)^{H}$ denote transpose and transpose conjugate, respectively. The trace of the matrix $\mathbf{M}$ is denoted by $\operatorname{Tr}(\mathbf{M})$. The mathematical expectation operator is denoted by $\mathbb{E}($.). The notation $\mathcal{N}(\underline{v}, \mathbf{M})$ stands for the complex multi-dimensional Gaussian random variable with mean $\underline{v}$, and covariance $\mathbf{M}$. Finally, the notation $(.)^{+}$denotes the function $\max (0,$.$) .$

\section{System model}

The global system under investigation is represented in Fig. 1. It consists of $K$ mobile terminals, and $S$ base stations using non-overlapping frequency bands (in Fig. $1, S=3$ ). Each mobile terminal has one single antenna, while the base station

\footnotetext{
${ }^{1}$ The optimum receiver would be implemented by a maximum likelihood (ML) decoder. However, in order to prove coding theorems, joint typicalitybased decoders are generally assumed, because they can be shown to be optimum for infinite sizes of codeword.
} 
can possibly have multiple antennas depending on the radio technology. The number of dimensions associated with base station $s \in\{1, \ldots, S\}$ is denoted by $N_{s}$. For example, if a CDMA system is used, $N_{s}$ represents the spreading factor; on the other hand, if the base station is equipped with multiple antennas, $N_{s}$ represents the number of receive antennas. Assuming time selective but frequency non-selective channels, ${ }^{2}$ the equivalent baseband signals received by the base stations can be written as

$$
\left\{\begin{array}{l}
\underline{y}_{1}(\tau)=\sqrt{\rho_{1}} \sum_{\ell=1}^{K} \underline{h}_{\ell, 1}(\tau) x_{\ell, 1}(\tau)+\underline{z}_{1}(\tau) \\
\underline{y}_{2}(\tau)=\sqrt{\rho_{2}} \sum_{\ell=1}^{K} \underline{h}_{\ell, 2}(\tau) x_{\ell, 2}(\tau)+\underline{z}_{2}(\tau) \\
\vdots \\
\underline{y}_{S}(\tau)=\sqrt{\rho_{S}} \sum_{\ell=1}^{K} \underline{h}_{\ell, S}(\tau) x_{\ell, S}(\tau)+\underline{z}_{S}(\tau),
\end{array}\right.
$$

where $\forall k \in\{1, \ldots, K\}, \forall s \in\{1, \ldots, S\}, x_{k, s}(\tau)$ is the signal transmitted by user $k$ to base station $s$ at time $\tau$, satisfying $\sum_{s=1}^{S} \mathbb{E}\left|x_{k, s}\right|^{2} \leq 1, \underline{h}_{k, s}(\tau)$ is the $N_{s}$-dimensional stationary, and zero-mean ergodic complex Gaussian channel vector associated with user $k$ for the system $s, \underline{z}_{s}(\tau)$ is an $N_{s}$-dimensional complex white Gaussian noise distributed as $\mathcal{N}\left(\underline{0}, n_{0} B_{s} \mathbf{I}\right)$, where $n_{0}$ is the receive noise power spectral density, $B_{s}$ the bandwidth of system $s, \rho_{s}$ is the signal-to-noise ratio (SNR) in system $s$, defined as $\rho_{s}=\frac{P}{n_{0} B_{s}}$, and $P$ is the transmit power available at a given terminal. For simplicity and clarity, we henceforth implicitly assume that the mobile terminals have the same transmit power, which is a reasonable assumption (see e.g. [29] for more information). Otherwise, the case with distinct transmit powers could be easily taken into account. For simplicity, we will omit the time index $\tau$ from our notations. In our analysis, the flat fading channel vectors of the different links can possibly vary from symbol vector (or space-time codeword) to symbol vector (or space-time codeword). We assume that the receivers (base stations) know their channel matrices (coherent communication assumption), and send the channel distribution information (CDI) through reliable links to a central controller. Knowing the channels of all users, the central controller implements the algorithm and indicates to each user how he has to share his transmit power between the different links. The transmitters, therefore, do not need any knowledge on the channels (neither channel state nor distribution information).

As we will consider the overall system sum-rate as the performance criterion, and assume a large system, in terms of both the number of users and dimensions at the base stations $\left(N_{1}, \ldots, N_{S}\right)$, it is convenient to rewrite the received signal in matrix form:

$$
\left\{\begin{array}{l}
\underline{y}_{1}=\sqrt{\rho_{1}} \mathbf{H}_{1} \underline{x}_{1}+\underline{z}_{1} \\
\underline{y}_{2}=\sqrt{\rho_{2}} \mathbf{H}_{2} \underline{x}_{2}+\underline{z}_{2} \\
\vdots \\
\underline{y}_{S}=\sqrt{\rho_{S}} \mathbf{H}_{S} \underline{x}_{S}+\underline{z}_{S},
\end{array}\right.
$$

where $\forall s \in\{1, \ldots, S\}, \mathbf{H}_{s}=\left[\underline{h}_{1, s} \ldots \underline{h}_{K, s}\right]$ and $\underline{x}_{s}=\left(x_{1, s}, \ldots, x_{K, s}\right)^{\mathrm{T}}$. We assume that the channel matrix of a given system can be factorized, in the sense of the Hadamard product, as a product of two matrices

$$
\mathbf{H}_{s}=\mathbf{G}_{s} \odot \mathbf{W}_{s},
$$

where $\mathbf{W}_{s}$ is the matrix of the instantaneous channel gains, which are assumed to be i.i.d zero-mean and unit variance, and $\mathbf{G}_{s}$ is the pattern mask specific to a given technology, containing the arbitrary variances of the elements of $\mathbf{H}_{s}$. This model is broad enough to incorporate several radio access technologies. Here are three typical examples:

- MIMO systems: $N_{s}$ represents the number of antennas at the base station $s$ and $K$ the number of users (each equipped with a single antenna). The matrices $\mathbf{W}_{s}$ and $\mathbf{G}_{s}$ are respectively an i.i.d. zero mean Gaussian matrix and a $N_{t} \times K$ correlation matrix. The Kronecker model $[1,4,23]$ is very used to model MIMO channels. It assumes that the channel transfer matrix can be factorized as $\mathbf{H}_{\mathbf{s}}=\mathbf{R}_{s}^{\frac{1}{2}} \boldsymbol{\Theta}_{s} \mathbf{T}_{s}^{\frac{1}{2}}$, where the matrices $\mathbf{T}_{s}$ and $\mathbf{R}_{s}$, respectively account for the possible correlation effects at the transmitter and receiver. What is important to note, is that in this context $g_{s}(i, j)=\sqrt{d_{i, s}^{(R)} d_{j, s}^{(T)}}$, where $d_{i, s}^{(R)}$ are the eigenvalues of the correlation matrix $\mathbf{R}_{s}$, at the receiver and $d_{j, s}^{(T)}$, are the eigenvalues of the correlation/path loss matrix at the transmitter $\mathbf{T}_{s}$;

- Flat fading CDMA systems: $N_{s}$ represents the spreading factor and $K$ the number of users. For a block fading channel, $\mathbf{W}_{s}$ and $\mathbf{G}_{s}$, are respectively the code matrix, where each column represents the code of a given user, and the channel gains matrix, where the columns are identical (due to the fact that we consider flat fading models);

\footnotetext{
2 The present information theoretic analysis can be directly extended to multipath channels (channels with memory). One can show that, provided that the maximum channel memory is negligible w.r.t. the size of the codewords used, the only difference is that one has to work in the frequency domain [17].
} 
- OFDM systems: $N_{s}$ represents the number of sub-carriers, and $K$ the number of users. Assuming for simplicity an OFDMA system, where each user uses one subcarrier, $\mathbf{W}_{s}$ and $\mathbf{G}_{s}$ are respectively an i.i.d. zero mean Gaussian matrix and the truncated identity matrix (as the channel matrices are not necessarily square). Note that if $K<N_{s}$, some sub-carriers are not used.

\section{Maximizing the sum-rate in the simplest scenario}

Here, we consider the simplest scenario, with 1 user and 1 dimension at each of the 2 base stations. The two frequency bands used by each of the cells are denoted by $B_{1}$ and $B_{2}$. For simplicity we assume that $B_{1}=B_{2}=\frac{B}{2}$. The scalar channels are assumed to be fixed here. This case is very simple, but captures some important features of the problem. The system of equations associated with the received signals is:

$$
\left\{\begin{array}{l}
y_{1}=\sqrt{\rho_{1}} h_{1} x_{1}+z_{1} \\
y_{2}=\sqrt{\rho_{2}} h_{2} x_{2}+z_{2}
\end{array}\right.
$$

The power fractions allocated to bands 1 and 2 are respectively denoted by $P_{1}=\alpha$ and $P_{2}=(1-\alpha)$, with $\alpha \in[0,1]$. We also introduce $\theta_{1}$ and $\theta_{2}$ defined by: $\theta_{1}=\frac{\left|h_{1}\right|^{2}}{\sigma^{2}}$ and $\theta_{2}=\frac{\left|h_{2}\right|^{2}}{\sigma^{2}}$, where $\sigma^{2}=\frac{n_{0} B}{2}$. The system sum-capacity can then be written as:

$$
\begin{aligned}
C & =\frac{B}{2} \max _{\alpha \in[0,1]}\left\{\log _{2}\left(1+\theta_{1} \alpha\right)+\log _{2}\left[1+\theta_{2}(1-\alpha)\right]\right\} \\
& =\frac{B}{2} \max _{\alpha \in[0,1]} \underbrace{\log _{2}\left\{1+\theta_{2}+\left[\theta_{1}-\theta_{2}+\theta_{1} \theta_{2}\right] \alpha-\theta_{1} \theta_{2} \alpha^{2}\right\}}_{R(\alpha)} .
\end{aligned}
$$

The function $\alpha \mapsto R(\alpha)$ is strictly concave, and its maximum is reached for:

$$
\alpha^{*}=\frac{1}{2}+\frac{1}{2}\left(\frac{1}{\theta_{2}}-\frac{1}{\theta_{1}}\right) \quad \text { whenever it belongs to }[0,1] .
$$

The power fraction allocated to system 1 is merely linear in the difference between the reciprocal of the receive signal-tonoise ratios associated with each of the systems. Three operating regimes can be distinguished:

- when $\theta_{1} \leq \frac{\theta_{2}}{1+\theta_{2}}: \alpha^{*}=0$; as we did not impose the function $R(\alpha)$ to meet the transmit power constraint, $\alpha^{*}=0$ translates the water-filling solution, when all the power has to be allocated to the dominant link (link 2 here), into the well known hard handover or best base station selection strategy. Symmetrically, when $\theta_{2} \leq \frac{\theta_{1}}{1+\theta_{1}}: \alpha^{*}=1$;

- when $\theta_{1}=\theta_{2}$ then the optimal solution is the uniform power allocation.

- for the other (non-negative) values for the pairs $\left(\theta_{1}, \theta_{2}\right)$ there generally exists a strictly better power allocation policy than the uniform PA and hard handover schemes.

\section{Large systems scenario analysis}

In this section, we consider a much more realistic scenario for wireless communications. The different links between transmitters and receivers are now block fading, and the numbers of users, systems and base station dimensions can be arbitrarily selected. Additionally, the base stations can have different bandwidths $B_{1}, \ldots, B_{S}$. The numbers of users and dimensions have to be large enough, in order to make our asymptotic analysis sufficiently accurate. More precisely, we consider a scenario where $K \rightarrow+\infty, \forall s \in\{1, \ldots, S\}, N_{s} \rightarrow+\infty$ with $\lim _{K \rightarrow \infty, N_{s} \rightarrow \infty} \frac{K}{N_{s}}=c_{s}$ and $0<c_{s}<+\infty$. However, it is now well-known that many asymptotic results from random matrix theory under the large system assumption apply for relatively small systems $[2,7,8]$.

Under these assumptions, our main objective is to derive the best power allocation scheme, in the sense of the sum-rate of the global system for different types of receivers. One can notice that the selected performance criterion is global, whereas power constraints are local, which is a key difference with the conventional power sharing problem between different subchannels.

\subsection{Optimum receiver}

When the optimum receiver is assumed at the base stations, maximizing the sum-rate leads to the Shannon sum-capacity of the global system. Considering the sum-rate point of the system, instead of an arbitrary operating point of the capacity region, has the advantage of simplifying the technical problem. In particular, considering the sum-rate as the performance criterion, allows us to exploit some results obtained for single-user fading MIMO (e.g. [28]). Note that the considered system consists of several orthogonal multiple access channels (MAC), with multi-dimensional receivers, and single-dimensional transmitters, under the assumption that CSIR (channel state information at the receiver) but no CDIT (CDI at the transmitter) 
is available. The sum-rate of each MAC is simply a special case of the general case analyzed by [32,33] for Rayleigh MIMO multiple access channels with input correlation with CSIR and CDIT (CDI at the transmitter). In our case, where the dimension of the signal transmitted by a terminal is one, the CDIT assumption amounts for a user to knowing its transmit power. By considering the system of (orthogonal) Eq. (2) the network ergodic sum-capacity per user can be expressed as:

$$
C=\max _{\mathbf{Q}_{1}, \ldots, \mathbf{Q}_{S}} \mathbb{E}\left[\frac{1}{K}\left(\sum_{s=1}^{S} B_{s} \log _{2}\left|\mathbf{I}+\rho_{s} \mathbf{H}_{s} \mathbf{Q}_{s} \mathbf{H}_{s}^{H}\right|\right)\right]
$$

where $\forall s \in\{1, \ldots, S\}, \mathbf{Q}_{s}=\mathbb{E}\left(\underline{\chi}_{s} \underline{x}_{s}^{H}\right)$. As long as the signals transmitted by the different users are independent, the matrices $\mathbf{Q}_{s}$ are diagonal: $\mathbf{Q}_{s}=\operatorname{Diag}\left(\alpha_{1, s}, \ldots, \alpha_{K, s}\right)$, where $\alpha_{k, s}$ denotes the fraction of its power user $k$ employs in system $s$. As the mobile terminals have identical transmit power, we have $\forall k \in\{1, \ldots, K\}, \sum_{s=1}^{S} \alpha_{k, s}=1$.

So far, we have not assumed anything about the numbers of users and base station dimensions. From now on, in order to simplify the optimization problem associated with Eq. (7) we will assume the asymptotic regime, as defined in the beginning of this section. Interestingly, in that case, an explicit equivalent for the network sum-rate can be obtained (from [13], see also [3]), whatever the pattern mask $\mathbf{G}_{s}$, as long as its continuous power profile, defined for $\left(\tau, \tau^{\prime}\right) \in[0,1]^{2}$ as $p_{N_{s}}\left(\tau, \tau^{\prime}\right)=g_{s}(i, j)$ with $\frac{i-1}{N_{s}} \leq \tau \leq \frac{i}{N_{s}}$ and $\frac{j-1}{N_{s} K} \leq \tau^{\prime} \leq \frac{j}{N_{s} K}$, converges uniformly to a bounded and piecewise continuous function as $N_{s} \rightarrow \infty$ [13], Corollary 10.1.2 in [12]. However, if the pattern mask is not structured at all, the expression of the large system equivalent can be quite complicated, and not always easy to exploit, whereas it is simpler for the class of separable channels (e.g. CDMA and MIMO channels). This is why we will mainly focus on this class of channel, while having in mind that the proposed framework can be extended to other technologies. Note that the OFDM case needs a separate treatment, since the power profile $p_{N_{s}}$ does not converge uniformly. However, it is not difficult to see that one can obtain the same capacity expression as in the separable case $[15,22,26-28]$ with classical techniques. Therefore, for at least the three aforementioned types of technologies, the constrained optimization under consideration can be simplified by finding a certain approximation $\tilde{C}$ of $C$, which can be obtained by exploiting the original results of [13,31] which have been applied by $[15,22,26-28]$ to fading single-user vector channels. This is stated through the following proposition.

Proposition 4.1 (Equivalent of the Network Sum-Rate). An equivalent of (7) in the asymptotic regime, i.e. when $K \rightarrow+\infty$, $\forall s \in\{1, \ldots, S\}, N_{s} \rightarrow+\infty$ with $\lim _{K \rightarrow \infty, N_{s} \rightarrow \infty} \frac{K}{N_{s}}=c_{s}$ and $0<c_{s}<+\infty$, is:

$$
\begin{aligned}
\tilde{C}= & \max _{\underline{\alpha}_{1}, \ldots, \underline{\alpha}_{K}} \frac{1}{K}\left[\sum_{s=1}^{S} \sum_{\ell=1}^{K} B_{s} \log _{2}\left(1+\gamma_{\ell, s} \alpha_{\ell, s} r_{s}\right)+\frac{1}{K} \sum_{s=1}^{S} \sum_{j=1}^{N_{s}} B_{s} \log _{2}\left(1+\beta_{j, s} q_{s}\right)\right. \\
& \left.-\sum_{s=1}^{S} B_{s} v_{s} q_{s} r_{s} \log _{2} e-\sum_{\ell=1}^{K} \lambda_{\ell}\left(\sum_{s=1}^{S} \alpha_{\ell, s}-1\right)\right]
\end{aligned}
$$

where $\forall \ell \in\{1, \ldots, K\}, \lambda_{\ell}$ is the Lagrange multiplier associated with the power constraint of user $\ell$, guaranteeing that the sum of power fractions over the different systems equals one. The expression of $v_{s}$ depends on the technology used by system s: $v_{s}=K \rho_{s}$ if $s$ denotes the index of a MIMO system; $v_{s}=\frac{K}{N_{s}} \rho_{s}$ if $s$ denotes the index of a CDMA system. In both cases the parameters $\left\{\left(q_{s}, r_{s}\right)\right\}_{s \in\{1, \ldots, S\}}$ are determined as the unique solution of the following system of equations:

$$
\left\{\begin{array}{l}
r_{s}=\frac{1}{K v_{s}} \sum_{j=1}^{N_{s}} \frac{\beta_{j, s}}{1+\beta_{j, s} q_{s}} \\
q_{s}=\frac{1}{K v_{s}} \sum_{\ell=1}^{K} \frac{\gamma_{\ell, s} \alpha_{\ell, s}}{1+\gamma_{\ell, s} \alpha_{\ell, s} r_{s}} .
\end{array}\right.
$$

$\mathbf{H}_{s}=\mathbf{R}_{s}^{\frac{1}{2}} \boldsymbol{\Theta}_{s} \mathbf{T}_{s}^{\frac{1}{2}}, \boldsymbol{\Theta}_{s}$ is a matrix with i.i.d entries with unit-variance, $\gamma_{\ell, s}=v_{s} d_{\ell, s}^{(T)}, d_{\ell, s}^{(T)}$ is the $\ell$ th eigenvalue of $\mathbf{T}_{s}, \beta_{j, s}=v_{s} d_{j, s}^{(R)}$, $d_{j, s}^{(R)}$ is the jth eigenvalue of $\mathbf{R}_{s}$. For the OFDM case, Eq. (8) holds with $r_{s}=\rho_{s}, q_{s}=0$ and $\gamma_{\ell, s}=g_{s}^{2}(\ell, \ell)$.

The proof directly follows from $[27,28]$ since in our case the channels are also separable. In order to better understand, and interpret the provided result and make this paper self contained, we provide a special case drawn from [26]: a single MIMO system with SNR $\rho, K$ inputs, $N$ outputs and neither transmit nor receive correlation. The approximate capacity per receive antenna can be written in this case: $\tilde{C}=\frac{1}{N} \sum_{i=1}^{K} \log _{2}[1+\rho \alpha(i) r]+\log _{2}\left(\frac{N}{K} r\right)-\frac{N}{K}\left(\frac{N}{K}-r\right) \log _{2} e$ where $r$ is determined through the following fixed point equation

$$
\left\{\begin{array}{l}
r=\frac{N}{K} \frac{1}{1+\rho q} \\
q=\frac{1}{K} \sum_{i=1}^{K} \frac{\alpha(i)}{1+\rho \alpha(i) r} .
\end{array}\right.
$$


Therefore, we see that the large system approximation roughly allows one to transform the exact capacity expression of the fast fading MIMO system into a sum of individual capacities, similarly to a parallel set of Gaussian sub-channels. Now let us go back to the general case. In order to find the optimum power allocation scheme we need to derivate the argument of the maximum in Eq. (8), which we refer to as $\tilde{R}\left(\underline{\alpha}_{1}, \ldots, \underline{\alpha}_{K}\right)$. Obviously for all $s \in\{1, \ldots, S\}, r_{s}$ and $q_{s}$ are functions of the parameters to be optimized i.e. $\alpha_{1, s}, \ldots, \alpha_{K, s}$. It turns out that the partial derivative with respect to $\alpha_{k, s}$, is the same as it would be if $r_{s}$ and $q_{s}$ were assumed to be independent of this parameter, which is the purpose of the following lemma.

Lemma 4.2 (Property of the Equivalent of the Network Sum-Rate). For all $(k, s) \in\{1, \ldots, K\} \times\{1, \ldots, S\}$, the derivative of the sum-rate approximation $\tilde{R}\left(\underline{\alpha}_{1}, \ldots, \underline{\alpha}_{K}\right)$ with respect to $\alpha_{k, s}$, is the same as that obtained when assuming $r_{s}$ and $q_{s}$ to be independent of $\alpha_{k, s}$.

This key property is proved in Appendix A. This property of the large dimension equivalent of the sum-rate is instrumental in the determination of the optimum PA policy, because it considerably simplifies the optimization procedure, and allows us to cope with the convergence issue of $r_{s}$ and $q_{s}$ towards strict constants as the numbers of users and dimensions grow. Based on this argument, the fact that $\left(\underline{\alpha}_{1}, \ldots, \underline{\alpha}_{K}\right) \mapsto \tilde{R}\left(\underline{\alpha}_{1}, \ldots, \underline{\alpha}_{K}\right)$ is a strictly concave function (its Hessian is strictly positive) and using the notation $B_{s}=b_{s} \times B$ (where $B=B_{1}+\cdots+B_{S}$ ) in order to use dimensionless quantities, one can show that the optimum power fractions are given by the following proposition.

Proposition 4.3 (Power Allocation for the Optimum Receiver). In the asymptotic regime, the optimum power fraction of user $k$ in system $s$ is:

$$
\alpha_{k, s}^{*}=\left[\frac{b_{s}}{\sum_{t \in s_{k}^{+}} b_{t}}\left(1+\sum_{t \in s_{k}^{+}} \frac{1}{\gamma_{k, t} r_{t}}\right)-\frac{1}{\gamma_{k, s} r_{s}}\right]^{+},
$$

where for each user $k$, the set $\delta_{k}^{+}$represents the systems/sub-channels which receive a non-zero power; $\left|\wp_{k}^{+}\right| \leq S$ by definition. User $k$ will allocate power to system $s$ if and only if the quantity $\frac{b_{s}}{\lambda_{k} \ln 2}-\frac{1}{\gamma_{k, s} r_{s}}$ is strictly positive.

We see that, thanks to the large system assumption, analysis of the general system under consideration (with fading and arbitrary numbers of users and base station dimensions), leads to a solution similar to that obtained for the elementary system of Eq. (6), where the channels were assumed to be static. Indeed, we also have a water-filling equation for the optimum power allocation scheme, which is due to the averaging effect induced by the large system assumption. Let us give one special case of Eq. (11): the case where the base stations have the same bandwidth (e.g. UMTS-FDD + UMTS-TDD base stations):

$$
\alpha_{k, s}^{*}=\left[\frac{1}{\left|\delta_{k}^{+}\right|}+\frac{1}{\left|\delta_{k}^{+}\right|} \sum_{t \in s_{k}^{+}} \frac{1}{\gamma_{k, t} r_{t}}-\frac{1}{\gamma_{k, s} r_{s}}\right]^{+} .
$$

Here can be seen even more clearly, in Eq. (12), that the obtained result can be linked to the water-filling power allocation derived for the elementary scenario Eq. (6): the optimum power fraction comprises a term corresponding to the uniform PA (i.e. the term $\frac{1}{\left|s_{k}^{+}\right|}$) plus a term that characterizes the difference of quality between the system under consideration $\left(\frac{1}{\gamma_{k, s} r_{s}}\right)$, and the average of all the systems $\left(\frac{1}{\left|s_{k}^{+}\right|} \sum_{t \in s_{k}^{+}} \frac{1}{\gamma_{k, t} r_{t}}\right)$.

The capacity of the system under consideration is achieved, if and only if all the water-filling equations Eq. (11) are verified simultaneously. This is obviously the case by construction of the derivation of the water-filling equations, and the convexity of the optimization region. The main issue to be mentioned now, is a way of implementing the proposed power allocation scheme. We propose an iterative algorithm to implement the optimal power allocation policy:

(1) Initialization: assume a uniform power allocation scheme i.e. $\forall(k, s) \in\{1, \ldots, K\} \times\{1, \ldots, S\}, \alpha_{k, s}=\frac{1}{s}$.

(2) Compute the corresponding value for $r_{s}$ by using the fixed-point method: the first equation of system (9) can be written in the form: $r_{s}=f_{s}\left(r_{s}\right)$.

(3) Iterate the procedure while the desired accuracy on the power fractions is not reached.

- For users $k \in\{1, \ldots, K\}$ :

. Update the power fractions by using the water-filling equation (11).

. Update the value of $r_{s}$.

A similar algorithm has been recently used by [8,9], in order to derive the capacity of single-user Rician MIMO channels with antenna correlation. Based on the results of $[8,9]$ one is ensured that the approximated ergodic mutual information is a strictly concave function of the transmit power fractions $\left\{\underline{\alpha}_{1}, \ldots, \underline{\alpha}_{K}\right\}$ and if the iterative power allocation algorithm converges, then it converges towards the global maximum. At each step of the iterative procedure, the total sum-rate of the system is therefore increasing and generally (all the simulations performed in $[8,9]$ and this paper confirmed this point) 
converges to a limit. At the limit, all power fractions will verify the water-filling equations. As already mentioned, the system sum-capacity would be achieved by using a maximum likelihood receiver at all the base stations. More pragmatically we now turn our attention to sub-optimum receiver structures, which can be implemented more easily in real systems. One of the questions we want to answer is whether the optimal PA, in terms of the network sum-rate, for other types of receivers can also be expressed through a simple water-filling equation.

\subsection{MMSE receiver}

The MMSE receiver is known to be the best linear multi-user receiver, in terms of signal-to-interference plus noise ratio (SINR). In our context, the MMSE receiver at base station $s \in\{1, \ldots, S\}$ for user $k \in\{1, \ldots, K\}$ can be written as:

$$
\underline{w}_{k, s}^{H}=\underline{h}_{k, s}^{H}\left(\sum_{\ell=1}^{K} \alpha_{\ell, s} \underline{h}_{\ell} \underline{h}_{\ell}^{H}+\sigma^{2} \mathbf{I}\right)^{-1},
$$

and the SINR is given by:

$$
\eta_{k, s}^{(m m s e)}=\alpha_{k, s} \underline{h}_{k, s}^{H}\left(\sum_{\ell=1, \ell \neq k}^{K} \alpha_{\ell, s} \underline{h}_{\ell} \underline{h}_{\ell}^{H}+\sigma^{2} \mathbf{I}\right)^{-1} \underline{h}_{k, s} .
$$

In order to express the sum-rate achieved by the overall system when the MMSE receiver is used at the base stations, one just needs to determine the SINR at the input of each MMSE receiver. It turns out that each of these SINRs converges to a limit, and is especially easy to express in the large dimensions regime (see e.g. [5,25]). Let $\tilde{\eta}_{\ell, s}^{(m m s e)}$ be the asymptotic SINR for user $\ell$ in the output of the MMSE receiver at base station $s$. The achievable approximate ergodic sum-rate is then given by:

$$
\tilde{R}_{\text {sum }}^{(m m s e)}=\mathbb{E}[\sum_{s=1}^{S} \sum_{\ell=1}^{K} \underbrace{\log _{2}\left(1+\tilde{\eta}_{\ell, s}^{(m m s e)}\right)}_{\tilde{R}_{k, s}^{(m m s e)}}] .
$$

The asymptotic SINR expression in the MMSE output can be shown to be (see e.g. [24,25]):

$$
\forall \ell \in\{1, \ldots, K\}, \quad \tilde{\eta}_{\ell, s}^{(m m s e)}=\frac{\alpha_{\ell, s}}{N_{s}} \sum_{i=1}^{N_{s}} \frac{g_{s}^{2}(i, \ell)}{\sigma^{2}+\frac{1}{N_{s}} \sum_{j \neq \ell}^{K} \frac{\alpha_{j, s} g_{s}^{2}(i, j)}{1+\tilde{\eta}_{j, s}^{(m m s e)}}} .
$$

To find the amount of power user $k$ has to allocate to system $s$ one needs to derivate the sum-rate (Eq. (15)) w.r.t. $\alpha_{k, s}$. Unlike the asymptotic sum-rate achieved by the optimum receiver, the asymptotic sum-rate achieved by using the MMSE receiver is not always a concave function of $\left(\underline{\alpha}_{1}, \ldots, \underline{\alpha}_{K}\right)$. In order to obtain an analytical solution (otherwise an exhaustive numerical optimization of the sum-rate can always be performed), and avoid using possibly computationally demanding numerical optimization techniques, we propose to approximate the asymptotic sum-rate by a concave function, by introducing the two approximations (given below). This leads to the following proposition.

Proposition 4.4 (Optimum Power Allocation for the MMSE Receiver). Assume that

(1) $\tilde{\eta}_{k, s}^{(m m s e)}=a_{k, s}^{(m m s e)} \times \alpha_{k, s}$ with $\frac{\partial a_{k, s}}{\partial \alpha_{k, s}}=0$;

(2) $\left|\frac{\partial \tilde{R}_{k, s}^{(m m s e)}}{\partial \alpha_{k, s}}\right| \gg\left|\sum_{\ell \neq k} \frac{\partial \tilde{R}_{\ell, s}^{(m m s e)}}{\partial \alpha_{k, s}}\right|$.

In the asymptotic regime, the optimum power fraction of user $k$ in system $s$ is:

$$
\alpha_{k, s}^{(m m s e)}=\left[\omega_{k}-\frac{1}{a_{k, s}^{(m m s e)}}\right]^{+}
$$

where $\omega_{k} \triangleq \frac{1}{\lambda_{k} \ln 2}$ is the water-level for user $k$ and

$$
a_{k, s}^{(m m s e)} \triangleq \frac{1}{N_{s}} \sum_{i=1}^{N_{s}} \frac{g_{s}^{2}(i, k)}{\sigma^{2}+\frac{1}{N_{s}} \sum_{j \neq k}^{K} \frac{\alpha_{j, s} g_{s}^{2}(i, j)}{1+\tilde{\eta}_{j, s}^{(m m s e)}}} .
$$


Proof. By setting the derivative of the constrained asymptotic sum-rate to zero, one directly obtains that:

$$
\begin{aligned}
& \frac{\partial}{\partial \alpha_{k, s}}\left[\tilde{R}_{\text {sum }}^{(m m s e)}-\sum_{\ell=1}^{K} \lambda_{\ell}\left(\sum_{s=1}^{S} \alpha_{\ell, s}-P\right)\right]=0 \\
& \Leftrightarrow \frac{1}{\ln 2} \frac{\frac{\partial \tilde{\eta}_{k, s}^{(m m s e)}}{\partial \alpha_{k, s}}}{1+\tilde{\eta}_{k, s}^{(m m s e)}}-\lambda_{k}=0 . \quad \square
\end{aligned}
$$

The validity of assumptions (1) and (2) is discussed in Appendix B and will also be commented on in the simulation part. The first assumption is actually exactly verified in the finite case, and we would also like its large system equivalent to have this property. The second assumption is motivated by the fact that in a many user network, the behavior of a single user should have almost no impact on the SINR of another user of this network. Mathematically, as the proof above shows, the motivations for assuming (1) and (2) is that the optimization problem becomes very similar to the one investigated for the optimum receiver. Therefore, like the optimum receiver, the approximate optimum power allocation policy is given by a simple water-filling equation.

\subsection{Matched filter}

Now we go a step further in decreasing receiver complexity. We assume a matched filter at all the base stations. The MF for user $k$ at base station $s$, simply consists in multiplying the received signal $\underline{y}_{s}$ by $\underline{h}_{k, s}^{H}$. The signal at the MF output is expressed as

$$
\underline{h}_{k, s}^{H} \underline{y}_{s}=\left\|\underline{h}_{k, s}\right\|^{2} x_{k, s}+\sum_{\ell \neq k} \underline{h}_{k, s}^{H} \underline{h}_{\ell, s} x_{\ell, s}+\underline{h}_{k, s}^{H} z_{k, s},
$$

and the corresponding SINR follows:

$$
\eta_{k, s}^{(m f)}=\frac{\left\|\underline{h}_{k, s}\right\|^{4} \alpha_{k, s}}{\sigma^{2}\left\|\underline{h}_{k, s}\right\|^{2}+\sum_{\ell \neq k} \alpha_{\ell, s}\left|\underline{h}_{k, s}^{H} \underline{h}_{\ell, s}\right|^{2}} .
$$

In the asymptotic regime the SINR becomes (see e.g. $[24,25]$ )

$$
\tilde{\eta}_{k, s}^{(m f)}=\frac{\alpha_{k, s}\left(\sum_{i=1}^{N_{s}} g_{s}^{2}(i, k)\right)^{2}}{\sigma^{2} N_{s} \sum_{i=1}^{N_{s}} g_{s}^{2}(i, k)+\sum_{\ell \neq k} \alpha_{\ell, s} \sum_{i=1}^{N_{s}} g_{s}^{2}(i, k) g_{s}^{2}(i, \ell)} .
$$

The asymptotic system sum-rate achieved by using the MF at the reception is:

$$
\tilde{R}_{\text {sum }}^{(m f)}=\mathbb{E}\left[\sum_{s=1}^{S} \sum_{\ell=1}^{K} \log _{2}\left(1+\tilde{\eta}_{\ell, s}^{(m f)}\right)\right] .
$$

The optimum power allocation for the marched filter is then given by the following proposition.

Proposition 4.5 (Optimum Power Allocation for the MF). Assume that $\left|\frac{\partial \tilde{R}_{k, s}^{(m m s e)}}{\partial \alpha_{k, s}}\right| \gg\left|\sum_{\ell \neq k} \frac{\partial \tilde{R}_{\ell, s}^{(m m s e)}}{\partial \alpha_{k, s}}\right|$. In the asymptotic regime, the optimum power fraction of user $k$ in system $s$ is:

$$
\alpha_{k, s}^{(m f)}=\left[\omega_{k}-\frac{1}{a_{k, s}^{(m f)}}\right]^{+},
$$

where

$$
a_{k, s}^{(m f)}=\frac{\left(\sum_{i=1}^{N_{s}} g_{s}^{2}(i, k)\right)^{2}}{\sigma^{2} N_{s} \sum_{i=1}^{N_{s}} g_{s}^{2}(i, k)+\sum_{\ell \neq k} \alpha_{\ell, s} \sum_{i=1}^{N_{s}} g_{s}^{2}(i, k) g_{s}^{2}(i, \ell)},
$$

and $\omega_{k} \triangleq \frac{1}{\lambda_{k} \ln 2}$ is the water-level for user $k$. 
Proof. A quick look at the sum-rate expression shows that the situation is similar to that encountered with the MMSE receiver. The only difference is that one does not need to introduce assumption (1) since the $\operatorname{SINR} \eta_{k, s}^{(m f)}$ is always proportional to $\alpha_{k, s}$, whatever the dimensions of the system. The stated result follows.

\section{Simulation example}

In all the simulations the following channel model will be assumed. The entries of $\mathbf{W}_{s}$ will be chosen to be i.i.d. with zero-mean and variance 1. For the CDMA case, the entries of $\mathbf{G}$ will be generated according to a Rayleigh distribution with variance 1, with independent columns and all the elements in each of them equal, corresponding to flat fading, and for MIMO a matrix of ones (no correlation). First we assume the optimum receiver at the base stations. We want to evaluate the performance gain brought by exploiting the available cross-system diversity, in comparison with the standard power allocation scheme (hard handover). For this, let us assume the following typical simulation setup in a cellular system: 50 active users $(K=50)$ and 4 CDMA base stations $(S=4)$ with different spreading factors $\left(\left(N_{1}, N_{2}, N_{3}, N_{4}\right)=(4,8,16,32)\right)$. Fig. B. 1 shows that for medium and high SNRs the performance loss induced by using only one technology at a time can be very significant, greater than $4 \mathrm{~dB}$ typically, which means that the mobile transmit power could be divided by a factor greater than 2 w.r.t. to the conventional strategy. On the other hand, for low SNRs, the hard handover solution performs better than the uniform PA, which shows the potential interest in implementing the optimum PA, which provides the best performance whatever the SNR. Also, in contrast to single-user MIMO systems, it can be seen that the gap in performance between uniform and optimum PA schemes does not shrink as the SNR increases. This observation has also been made in other simulation scenarios. Fig. B.2 shows a scenario with the same parameters as the one just analyzed, but now both CDMA and MIMO systems are considered, obtaining relatively similar results. In all the tested scenarios, the convergence of the proposed iterative power allocation algorithm was obtained after at most 10 iterations; note that the algorithm is said to have converged if the optimum power fractions are determined with an accuracy of $10^{-4}$.

Now we assume the simplest receiver at the base stations, namely the matched filter. There are two base stations and two users. The BS are equipped with multiple antennas: $N_{1}=2, N_{2}=4$. Fig. B.3 shows the network sum-rate achieved by using the MF for four different PA schemes: the optimum PA obtained by an exhaustive numerical search, the approximate PA obtained by assuming the two hypotheses stated in Sections 4.2 and 4.3, the uniform PA scheme and the hard handover. First, the figure shows that the corresponding approximation of the sum-rate is not very good, but it still provides a performance gain over the other PA schemes. Second, this simulation confirms that the uniform PA becomes more and more suboptimal w.r.t. to the exact optimum PA, as the SNR increases. Third, we clearly see that handover based PA suffers from a significant performance loss for medium and high SNRs. To sum up, we can say that, as a rule of thumb, the uniform PA can always be used and will provide significant gain with the advantage of being very simple to implement (no feedback mechanism required in particular).

The last figure, i.e. Fig. B.4 sums up the network performance for the three receivers investigated in this paper in the typical scenario $K=20, S=3,\left(N_{1}, N_{2}, N_{3}\right)=(4,8,32)$. It allows one to better evaluate the benefits from using the optimum receiver over the MMSE receiver and MF. Typical information that can be drawn from this figure is as follows: by simply using a MMSE receiver with uniform PA instead of the MF with hard handover (as used in current networks) a huge performance gain could be obtained by exploiting the available cross-system diversity. Of course, this comment holds for medium and high SNRs. If the network is also likely to operate in the low SNR regime, the optimum PA should be used, or a SNR-based switching mechanism between the hard handover and uniform PA could be introduced.

\section{Conclusions}

In this contribution, a cross-system power allocation algorithm has been provided in the context of MIMO, CDMA and OFDM technologies, in order to exploit the available cross-system diversity. Interestingly, in the asymptotic regime, a radio access technology can be characterized, from the information-theoretic point of view, by only a few parameters. Indeed, the solution for all the receivers turns out to be dependent only on a limited number of parameters: the dimensions of the system, number of users, channel gains, path loss, noise variance and correlation at the transmitter and the receiver.

As a consequence, for the optimum receiver, a simple cross-layer algorithm, analogous to the water-filling algorithm, can be implemented at the central controller to schedule the powers of all the users, in order to maximize the network capacity, and this can be done in a simple, iterative way, which generally converges to the optimum.

For MF and MMSE receivers, a water-filling solution can still be obtained by introducing two additional assumptions, which simplify the optimization problem, but at the price of performance loss that has to be evaluated in the situations of interest. For the typical scenarios considered in this paper, we saw that they were reasonable. The potential performance gain of cross-system diversity was shown to be important in several typical simulation setups. For instance, by simply using MMSE receivers at the base stations, and uniform PA over the different systems, the mobile transmit power could be divided by a factor greater than 10 with respect to a standard network, using the MF and hard handover PA scheme.

The proposed work could be extended by considering the outage probability, in order to further analyze the benefits of cross-system diversity, which will allow one to complete our comparisons between the hard handover, uniform and optimum PA schemes. It would also be interesting to study a more heterogeneous network, for instance by introducing 
CDMA base stations with multiple antennas and exploiting the results derived by [16]. As mentioned in this paper, more technologies can be considered since the condition on the patter mask matrices $\mathbf{G}_{s}$ are mild and the strong results of [13] can be directly applied in the proposed framework.

To conclude this paper, the authors want to stress the fact that our approach is (information) theoretical and aims at giving insight to researchers and network designers on a quite difficult problem. Obviously, many issues would need to be addressed to implement the proposed power allocation schemes. The way of coordinating base stations using different technologies is just one example of this kind of issues.

\section{Appendix A. Proof of Lemma 4.2}

We want to derivate the argument of the maximum in Eq. (8) with respect to $\alpha_{k, s}$. First note from the system of Eq. (9) that $r_{t}$ and $q_{t}$ do not depend on $\alpha_{k, s}$ for all $t \neq s$. Based on this observation one just needs to consider the following auxiliary function:

$$
\phi\left(\alpha_{k, s}\right)=\log _{2}\left\{\prod_{\ell=1}^{K}\left[1+\gamma_{\ell} \alpha_{\ell, s} r\left(\alpha_{k}\right)\right] \times \prod_{j=1}^{N}\left(1+\rho d_{j}^{2} q\left(\alpha_{k, s}\right)\right) \times \mathrm{e}^{-K \rho r\left(\alpha_{k, s}\right) q\left(\alpha_{k, s}\right)}\right\}
$$

where we dropped the system index $s$ and receiver subscript $(R)$ for sake of clarity.

Define $u \triangleq \prod_{\ell=1}^{K}\left[1+\gamma_{\ell} \alpha_{\ell, s} r\left(\alpha_{k, s}\right)\right]$ and $v \triangleq \prod_{j=1}^{N}\left(1+\rho d_{j}^{2} q\left(\alpha_{k, s}\right)\right) \times \mathrm{e}^{-K \rho r\left(\alpha_{k, s}\right) q\left(\alpha_{k, s}\right)}$.

With these notations:

$$
\frac{\partial \phi\left(\alpha_{k, s}\right)}{\partial \alpha_{k, s}}=\frac{1}{\ln 2} \frac{1}{u v} \frac{\partial u v}{\partial \alpha_{k, s}} .
$$

It turns out that $\frac{\partial(u v)}{\partial \alpha_{k}}=u v \times \frac{\gamma_{k} r}{1+\gamma_{k} \alpha_{k, s}}$. This is what we want to show.

We want to derivate the function $u$ w.r.t. $\alpha_{k, s}$. As $u$ is a product of functions $u_{\ell}$, i.e. $u=\prod_{\ell=1}^{K} u_{\ell}$, its derivative $u^{\prime}$ can be written as $u^{\prime}=u \times \sum_{\ell=1}^{K} \frac{u_{\ell}^{\prime}}{u_{\ell}}$ where

$$
u_{\ell}^{\prime}=\mid \begin{array}{ll}
\gamma_{\ell} \alpha_{\ell, s} r^{\prime} & \text { if } \ell \neq k \\
\gamma_{k}\left(r+\alpha_{k, s} r^{\prime}\right) & \text { if } \ell=k
\end{array}
$$

Using a similar reasoning for $v$ one can check that

$$
v^{\prime}=v \times\left[\sum_{j=1}^{N} \frac{\rho d_{j}^{2} q^{\prime}}{1+\rho d_{j}^{2} q}-K \rho\left(q^{\prime} r+q r^{\prime}\right)\right] .
$$

Now using the relations proved in the previous steps we have that

$$
\frac{\partial(u v)}{\partial \alpha_{k, s}}=u v \times \underbrace{\left(\sum_{\ell=1}^{K} \frac{u_{\ell}^{\prime}}{1+\gamma_{\ell} \alpha_{\ell, s} r}+\sum_{j=1}^{N} \frac{\rho d_{j}^{2} q^{\prime}}{1+\rho d_{j}^{2} q}-K \rho\left(q^{\prime} r+q r^{\prime}\right)\right)}_{\psi}
$$

with $\psi$ expanding as

$$
\psi=\sum_{\ell \neq k} \frac{\gamma_{\ell} \alpha_{\ell, s} r^{\prime}}{1+\gamma_{\ell} \alpha_{\ell, s} r}+\frac{\gamma_{k}\left(r+\alpha_{k, s} r^{\prime}\right)}{1+\gamma_{k} \alpha_{k, s} r}+\sum_{j=1}^{N} \frac{\rho d_{j}^{2} q^{\prime}}{1+\rho d_{j}^{2} q}-K \rho\left(q^{\prime} r+q r^{\prime}\right) .
$$

Now by observing that

$$
\left\{\begin{array}{l}
\sum_{\ell \neq k} \frac{\gamma_{\ell} \alpha_{\ell, s} r^{\prime}}{1+\gamma_{\ell} \alpha_{\ell, s} r}=\left(K \rho q-\frac{\gamma_{k} \alpha_{k}}{1+\gamma_{k} \alpha_{k, s} r}\right) r^{\prime} \\
\sum_{j=1}^{N} \frac{\rho d_{j}^{2} q^{\prime}}{1+\rho d_{j}^{2} q}=K \rho q^{\prime} r
\end{array}\right.
$$

we find that

$$
\psi=\frac{\gamma_{k} r}{1+\gamma_{k} \alpha_{k, s} r},
$$

which concludes the proof. 


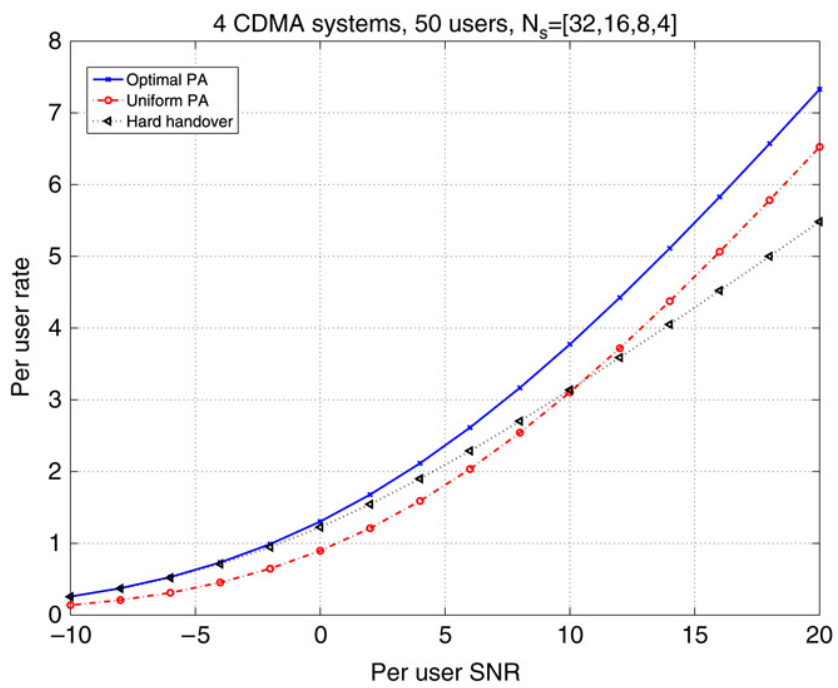

Fig. B.1. Optimal receiver. Performance gains brought by cross-system diversity (CDMA systems).

\section{Appendix B. Approximating the asymptotic system sum-rate by a concave function}

\section{B.1. The case of the MMSE receiver}

For the user of interest (i.e. user $k$ ):

$$
\frac{\partial \tilde{\eta}_{k, s}^{(\text {mmse })}}{\partial \alpha_{k, s}}=\frac{1}{N_{s}} \sum_{i=1}^{N_{s}}\left\{\frac{g_{s}^{2}(i, k)}{\sigma^{2}+\frac{1}{N_{s}} \sum_{j \neq k}^{K} \frac{\alpha_{j, s} g_{s}^{2}(i, j)}{1+\tilde{\eta}_{j, s}^{(m s)}}} \times\left[\sigma^{2}+\frac{1}{N_{s}} \sum_{j \neq k}^{K} \frac{\alpha_{j, s} g_{s}^{2}(i, j)}{1+\tilde{\eta}_{j, s}^{(m m s e)}}\left(1+\alpha_{k, s} \frac{\partial \tilde{\eta}_{j, s}^{(m m s e)}}{\partial \alpha_{k, s}} \frac{1}{1+\tilde{\eta}_{j, s}^{(m m s e)}}\right)\right]\right\}
$$

For all $\ell \neq k$,

$$
\frac{\partial \tilde{\eta}_{\ell, s}^{(m m s e)}}{\partial \alpha_{k, s}}=-\frac{\alpha_{\ell, s}}{N_{s}} \sum_{i=1}^{N_{s}}\left\{g_{s}^{2}(i, \ell) \times \frac{\frac{1}{N_{s}} \frac{g_{s}^{2}(i, k)}{1+\tilde{\eta}_{k, s}^{(m s e)}}+\frac{1}{N_{s}} \sum_{j \neq \ell} \alpha_{j, s} g_{s}^{2}(i, j)\left(\frac{-\partial \tilde{\eta}_{j, s}^{(m m s e)}}{\partial \alpha_{k, s}}\right) \frac{1}{\left(1+\tilde{\eta}_{j, s}^{(m m s e)}\right)^{2}}}{\left(\sigma^{2}+\frac{1}{N_{s}} \sum_{j \neq \ell} \frac{\alpha_{j, s} g_{s}^{2}(i, j)}{1+\tilde{\eta}_{j, s}^{(m m s e)}}\right)^{2}}\right\} .
$$

Let $\left|\tilde{\eta}_{M}^{\prime}\right|$ and $g_{M}$ be the maxima of $\left|\frac{\partial \tilde{\eta}_{\ell, s}^{(m m s e)}}{\partial \alpha_{k, s}}\right|$ and $g_{s}(i, \ell)$ over all the triplets $(i, \ell, s)$. By definition $\left|\frac{\partial \tilde{n}_{\ell, s}^{(m m s e)}}{\partial \alpha_{k, s}}\right| \leq\left|\tilde{\eta}_{M}^{\prime}\right|$. In fact, under reasonable assumptions, one can tighten this bound, this is the purpose of what follows. The main point is to assume that the entries $g_{s}(i, j)$ take finite values and do not vanish. Note that for MIMO systems the entries of the mask matrix $g_{s}(i, j)$ are effectively bounded, and they do not scale with $N_{s}$. However, for CDMA and OFDM systems, this is not true since for both cases, they represent the realizations of the channel impulse. As a Rayleigh distribution is assumed for the channel gains, they are not bounded mathematically. However, many works applying random matrix theory (see e.g. [6]) assume that the channel has a compact support. In practice, for physical reasons, the channel gains do not strictly vanish, and stay effectively in a finite interval, and therefore the proposed assumption makes sense.

For all $(k, s)$ in $\{1, \ldots, K\} \times\{1, \ldots, S\}$ one can easily check that

$$
\begin{aligned}
\left|\frac{\partial \tilde{\eta}_{\ell, s}^{(m m s e)}}{\partial \alpha_{k, s}}\right| & \leq \frac{\alpha_{\ell}}{N_{s}} \sum_{i=1}^{N_{s}} \frac{g_{s}^{2}(i, \ell)}{N_{s}} \sum_{j \neq \ell} g_{s}^{2}(i, j) \frac{\alpha_{j}}{\left(1+\tilde{\eta}_{j, s}^{(m m s e)}\right)^{2}}\left|\frac{\partial \tilde{\eta}_{j, s}^{(m m s e)}}{\partial \alpha_{k, s}}\right| \times \frac{1}{\sigma^{4}} \\
& \leq \frac{1}{N_{s}^{2}} \frac{g_{M}^{4}}{\sigma^{4}} \sum_{i=1}^{N_{s}} \sum_{j \neq \ell}\left|\frac{\partial \tilde{\eta}_{j, s}^{(m m s e)}}{\partial \alpha_{k, s}}\right| \\
& \leq\left(\frac{g_{M}}{\sigma}\right)^{4} \frac{K}{N}\left|\tilde{\eta}_{M}^{\prime}\right| .
\end{aligned}
$$




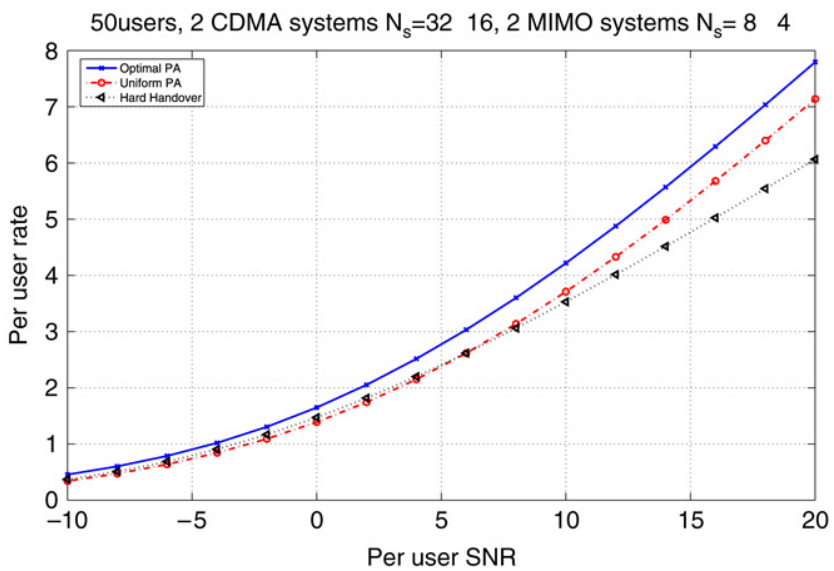

Fig. B.2. Optimal receiver. Performance gains brought by cross-system diversity (CDMA and MIMO systems).

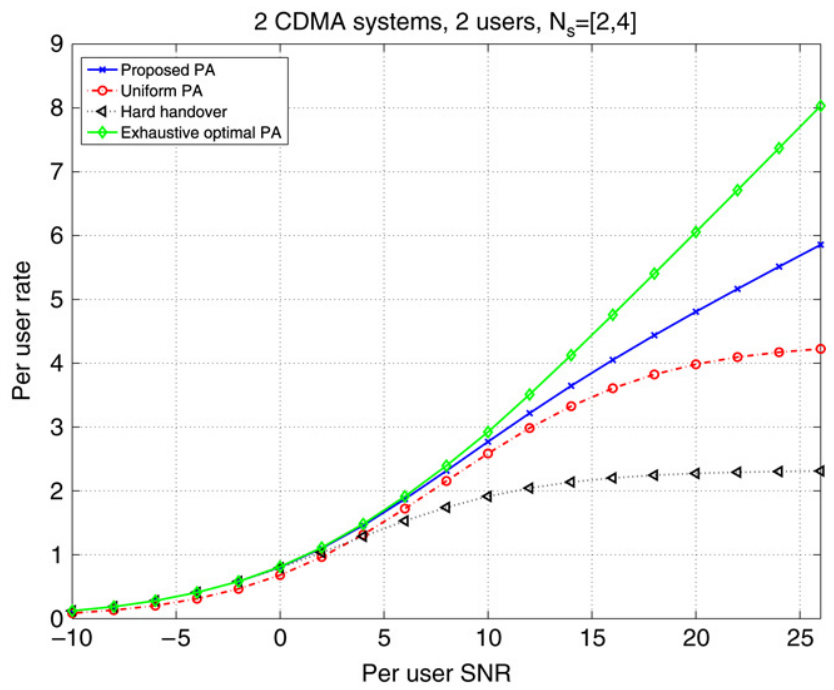

Fig. B.3. MF performance for the optimum PA, approximate optimum PA, uniform PA and hard handover PA.

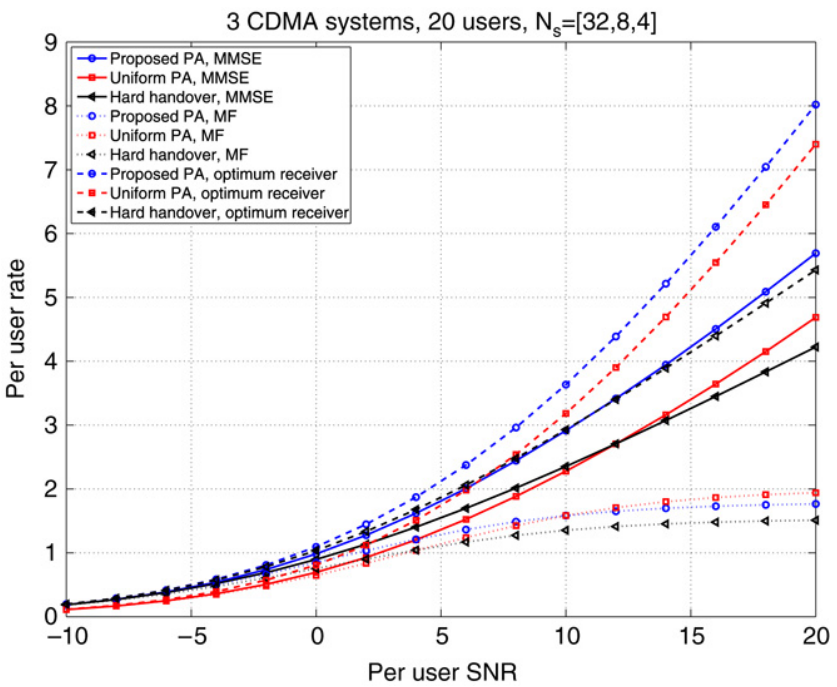

Fig. B.4. Optimum receiver vs MMSE receiver vs MF. 
Therefore we see that a sufficient condition for the MMSE output SINR of user $\ell$ to be considered as independent of the power allocation of user $k \neq \ell$, is that the ratio $\frac{K}{N}$ has to be small. Under this sufficient but not necessary condition, the approximate SINR $\tilde{\eta}_{k, s}$ can be considered to be proportional to $\alpha_{k, s}$ (Assumption (1)). For the second assumption to hold a sufficient but stronger condition is that the quantity $\frac{K^{2}}{N}$ is small. We therefore see that the validity of the proposed assumptions depends on the scenario under consideration.

\section{B.2. The case of the matched filter}

First, note that Assumption (1) is exactly verified both in the finite and large dimensions settings. So, here we focus on the validity of Assumption (2). In a given system $s$, we have

$$
\frac{\partial \tilde{R}_{s}^{(m f)}}{\partial \alpha_{k, s}} \triangleq \frac{\partial}{\partial \alpha_{k, s}} \sum_{\ell=1}^{K} \log _{2}\left(1+\tilde{\eta}_{\ell, s}^{(m f)}\right)=\frac{1}{\ln 2} \sum_{\ell=1}^{K} \frac{\partial \tilde{\eta}_{\ell, s}^{(m f)}}{\partial \alpha_{k, s}} \frac{1}{1+\tilde{\eta}_{\ell, s}^{(m f)}}
$$

with

$$
\left\{\begin{array}{l}
\frac{\partial \tilde{\eta}_{k, s}^{(m f)}}{\partial \alpha_{k, s}}=\frac{\tilde{\eta}_{k, s}^{(m f)}}{\alpha_{k, s}} \\
\frac{\partial \tilde{\eta}_{\ell, s}^{(m f)}}{\partial \alpha_{k, s}}=-\tilde{\eta}_{\ell, s}^{(m f)} \frac{\sum_{i=1}^{N_{s}} g_{s}^{2}(i, k) g_{s}^{2}(i, \ell)}{\sigma^{2} N_{s} \sum_{i=1}^{N_{s}} g_{s}^{2}(i, \ell)+\sum_{j \neq \ell} \alpha_{j, s} \sum_{i=1}^{N_{s}} g_{s}^{2}(i, \ell) g_{s}^{2}(i, j)}
\end{array} \text { for all } \ell \neq k .\right.
$$

Define $g_{M}^{2}=\max _{(\ell, s, i)} g_{s}^{2}(i, \ell)$ and $g_{m}^{2}=\min _{(\ell, s, i)} g_{s}^{2}(i, \ell)$ and upper bound the quantity of interest that is

$$
\begin{aligned}
\left|\sum_{\ell \neq k} \frac{\partial \tilde{R}_{\ell, s}}{\partial \alpha_{k, s}}\right| & =\left|\frac{1}{\ln 2} \sum_{\ell=1}^{K} \frac{\partial \tilde{\eta}_{\ell, s}^{(m f)}}{\partial \alpha_{k, s}} \frac{1}{1+\tilde{\eta}_{\ell, s}^{(m f)}}\right| \\
& \leq \frac{1}{\ln 2} \sum_{\ell \neq k}\left|\frac{\partial \tilde{\eta}_{\ell, s}^{(m f)}}{\partial \alpha_{k, s}}\right| \\
& \leq \frac{1}{\ln 2} \sum_{\ell \neq k}\left|\tilde{\eta}_{l, s}^{(m f)}\right| \frac{\sum_{i=1}^{N_{s}} g_{s}^{2}(i, k) g_{s}^{2}(i, \ell)}{\sigma^{2} N_{s} \sum_{i=1}^{N_{s}} g_{s}^{2}(i, \ell)} \\
& \leq \frac{1}{\ln 2} \sum_{\ell \neq k}\left|\tilde{\eta}_{l, s}^{(m f)}\right| \frac{N_{s} g_{M}^{4}}{\sigma^{2} N_{s} \sum_{i=1}^{N_{s}} g_{s}^{2}(i, \ell)} .
\end{aligned}
$$

At this point we have to distinguish between MIMO systems on the one hand, and CDMA and OFDM systems on the other hand. For MIMO systems we know that $\sum_{i=1}^{N_{s}} g_{s}^{2}(i, \ell) \geq N_{s} g_{m}^{2}$ where $g_{m}$ is finite, and different from zero. For CDMA and OFDM systems, as the channel realizations are into play, we exploit the central limit theorem, which allows us to write $\sum_{i=1}^{N_{s}} g_{s}^{2}(i, \ell)=N_{s}\left(\mu+o\left(\frac{1}{\sqrt{N_{s}}}\right)\right)$ where $\mu$ is the average energy of the channel gain (assumed to be normalized to one). In any case, the sum of interest can be bounded by const. $\times \frac{K}{N_{s}}$, which gives us a sufficient condition in order for Assumption 2 to hold for the matched filter.

\section{References}

[1] P. Almers, et al., Survey of channel and radio propagation models for wireless MIMO systems, EURASIP Journal on Wireless Communications and Networking 2007 (2007) doi:10.1155/2007/19070. Article ID 19070, 19 pages.

[2] E. Biglieri, G. Taricco, A. Tulino, How far is infinity? Using asymptotic analyses in multiple-antennas systems, in: Proc. IEEE Int. Symp. Spread Spectrum Techniques and Applications, Prague, Czech Republic, 2002, pp. 1-6.

[3] N. Bonneau, M. Debbah, E. Altman, A. Hjorungnes, Non-Atomic games for multi-user systems, in: Game Theory in Communication Systems, IEEE J. Sel. Areas Commun. (in press). To appear 4th quarter of 2008.

[4] C.-N. Chuah, D. Tse, J.M. Kahn, R. Valenzuela, Capacity scaling in MIMO wireless systems under correlated fading, IEEE Trans. Inform. Theory 48 (3) (2002) 637-650.

[5] M. Debbah, Linear precoders for OFDM wireless communications, Ph.D. Dissertation, École Normale Supérieure, Cachan, June 2002.

[6] M. Debbah, W. Hachem, P. Loubaton, M. de Courville, MMSE analysis of certain large isometric random precoded systems, IEEE Trans. Inform. Theory 49 (5) (2003) 1293-1311.

[7] J. Dumont, P. Loubaton, S. Lasaulce, M. Debbah, On the asymptotic performance of MIMO correlated Rician channels, in: Proc. IEEE Int. Conf. Acoustics, Speech, and Signal Processing, vol. 5, Philadelphia, PA, 2005, pp. 813-816. 
[8] J. Dumont, P. Loubaton, S. Lasaulce, On the capacity achieving transmit covariance matrices of MIMO correlated Rician channels: a large system approach, in: Proc. IEEE Global Telecommunications Conf., San Francisco, CA, 2006, pp. 1-6.

[9] J. Dumont, W. Hachem, S. Lasaulce, P. Loubaton, J. Najim, On the capacity achieving covariance matrix of Rician MIMO channels: An asymptotic approach, IEEE Trans. Inform. Theory, available on the Arxiv website at: http://front.math.ucdavis.edu/0710.4051 (submitted for publication).

[10] H. Feng, W. Furong, A Cost Sensitive Best Network Selection Scheme in Heterogeneous Wireless Networks, in: Proc. of the Int. Conf. on Wireless Communications, Networking and Mobile Computing, Austin, TX, 2007, pp. 1753-1756.

[11] B. Fette, Cognitive Radio Technology, Newnes editors, 2006.

[12] V.L. Girko, Theory of Random Determinants, Kluwer, Norwell, MA, 1990.

[13] V.L. Girko, Theory of Stochastic Canonical Equations, vol. 1 and vol. 2, Kluwer, Dordrecht, The Netherlands, 2001.

[14] S. Grech, P. Eronen, Implications of unlicensed mobile access (UMA) for GSM security, in: Proc. IEEE Security and Privacy for Emerging Areas in Communications Networks Conf., Athens, Greece, 2005, pp. 3-12.

[15] W. Hachem, O. Khorunzhiy, P. Loubaton, J. Najim, L. Pastur, A new approach for capacity analysis of large dimensional multi-antenna channels, IEEE Trans. Inform. Theory (in press).

[16] S. Hanly, D. Tse, Resource pooling and effective bandwidths in CDMA systems with multiuser receivers and spatial diversity, IEEE Trans. Inform. Theory 47 (4) (2001) 1328-1351.

[17] W. Hirt, J. Massey, Capacity of discrete-time Gaussian channels with intersymbol interference, IEEE Trans. Inform. Theory 34 (3) (1988) $380-388$.

[18] K. Kim, Y. Han, S-L. Kim, Joint subcarrier and power allocation in uplink OFDMA systems, IEEE Commun. Lett. 9 (6) (2005) 526-528.

[19] K.-C. Lee, G. Karmi, B. Mohanty, T.R. Sutton, N.A. Ziv, Inter-system calling supporting inter-system soft handoff, United States Patent 5940762, August $17,1999$.

[20] J. Mitola, et al., Cognitive radios: Making software radios more personal, IEEE Pers. Commun. 6 (4) (1999) 13-18.

[21] D. Molony, Fixed-mobile integration, Commun. Week (1998) 23-27.

[22] A.L. Moustakas, S.H. Simon, A.M. Sengupta, MIMO capacity through correlated channels in the presence of correlated interferers and noise: A (not so) large N analysis, IEEE Trans. Inform. Theory 49 (10) (2003) 2545-2561.

[23] D.S. Shiu, G.J. Foschini, M.J. Gans, J.M. Kahn, Fading correlation and its effects on the capacity of multielement antenna systems, IEEE Trans. Commun. 48 (3) (2000) 502-513.

[24] A. Suárez, R.L. de Lacerda, M. Debbah, Linh-Trung Nguyen, Power allocation under quality of service constraints for uplink multi-user MIMO systems, in: Proc. 10th IEEE Biennial Vietnam Conf. Radio \& Electronics, Hanoi, Vietnam, 2006.

[25] A. Suárez, M. Debbah, L. Cottatellucci, E. Altman, Optimal decoding order under target rate constraints, in: Proc. 8th IEEE Int. Workshop on Signal Processing Advances for Wireless Communications, Helsinki, Finland, 2007, pp. 1-5.

[26] A. Tulino, A. Lozano, S. Verdú, Capacity-achieving input covariance for correlated multi-antenna channels, in: Proc. of the 41th Annual Allerton Conf. on Communications, Control and Computing, Monticello, IL, 2003.

[27] A. Tulino, S. Verdú, Random matrices and wireless communications, Foundations and trends in communications and information theory, NOW, The Essence of Knowledge, 2004.

[28] A.M. Tulino, A. Lozano, S. Verdú, Impact of antenna correlation on the capacity of multi-antenna channels, IEEE Trans. Inform. Theory 51 (7) (2005) 2491-2509.

[29] UMTS World Association http://www.umtsworld.com/technology/technology.htm.

[30] M. Vrdoljak, S. Ivan Vrdoljak, G. Skugor, Fixed-mobile convergence strategy: Technologies and market opportunities, IEEE Commun. Mag. (2000) $116-121$.

[31] J.W. Silverstein, Z.D. Bai, On the empirical distribution of eigenvalues of a class of large dimensional random matrices, J. Multivariate Anal. 54 (1995) $175-192$.

[32] A. Soysal, S. Ulukus, Optimum power allocation for single-user MIMO and multi-user MIMO-MAC with partial CSI, IEEE J. Sel. Areas. Commun. 25 (7) (2007) 1402-1412.

[33] A. Soysal, S. Ulukus, Optimality of beamforming in fading MIMO multiple access channels, IEEE Trans. Commun. (submitted for publication).

[34] H.J. Wang, R.H. Katz, J. Giese, Policy-enabled handoffs across heterogeneous wireless networks, in: Proc. 2nd IEEE Workshop on Mobile Computing Systems and Applications, New Orleans, LA, 1999, pp. 51-60.

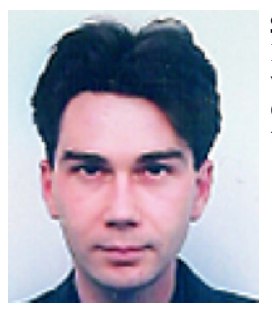

Samson Lasaulce is Chargé de Recherche CNRS at Supélec (LSS). He is a former student from École Normale Supérieure and École Nationale Supérieure de Télécommunications. He has been working with Motorola Labs for three years (1999, 2000, 2001) and with France Télécom R\&D for two years (2002, 2003). Since 2004, he has joined the CNRS and Supélec. Since 2004, he is also Chargé d'Enseignement at École Polytechnique. His broad interests lie in the areas of communications, signal processing and information theory with a special emphasis on game theory for wireless communications.

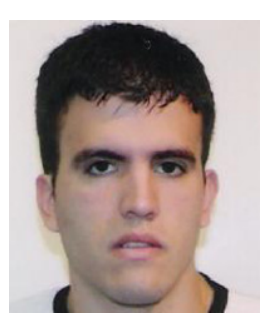

Alberto Suárez was born in Oviedo, Spain, in 1983. He did his telecommunication engineering studies in the Universidad de Oviedo. In 2006 he obtained the M.Sc degree in Digital Communications and Signal Processing from the Université de Nice, France. In October 2006 he joined the Mobile Communications department of Eurecom, Sophia Antipolis, France, where he is currently working towards the Ph.D. degree. His research interests lie in the topics of wireless communications and information theory.

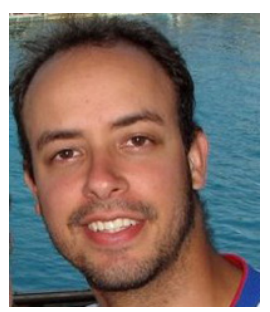

Raul de Lacerda was born in 1981 in Fortaleza/Ceara, Brazil. He entered the Federal University of Ceará (UFC) in 1999 where he obtained the B.Sc. and M.Sc. telecom engineering degrees respectively in 2003 and 2005. In 2004, he went to France, where he entered the University of Nice and obtained a second M.Sc degree (DEA SiCom/STIC - Signal and Digital Communications). Since October 2005, he has been with the Mobile Departament at Eurecom under the supervision of Prof. Mérouane Debbah/Supelec and Prof. João C.M. Mota/UFC. 


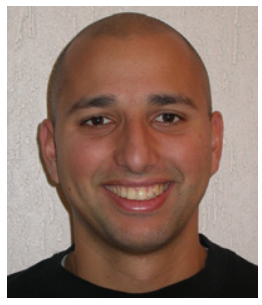

Mérouane Debbah was born in Madrid, Spain. He entered the Ecole Normale Supérieure de Cachan (France) in 1996 where he received the M.Sc and the Ph.D. degrees respectively in 1999 and 2002. From 1999 to 2002, he worked for Motorola Labs on Wireless Local Area Networks and prospective fourth generation systems. From 2002 until 2003, he was appointed Senior Researcher at the Vienna Research Center for Telecommunications (FTW) (Vienna, Austria) working on MIMO wireless channel modeling issues. From 2003 until 2007, he joined the Mobile Communications department of the Institute Eurecom (Sophia Antipolis, France) as an Assistant Professor. He is presently a Professor at Supelec (Gif-sur-Yvette, France), holder of the AlcatelLucent Chair on Flexible Radio. His research interests are in information theory, signal processing and wireless communications. Mérouane Debbah is the recipient of the 2007 Globecom best paper award as well as the Valuetools 2007 best student paper award. 